\title{
CORRELATION OF WIDTH OF ZONE OF KERATINIZED TISSUE AND GINGIVAL TISSUE THICKNESS WITH PERIODONTAL STATUS IN ANTERIOR TEETH
}

\author{
Gautami Subhadra Penmetsa1, Marella Sai Supriya², Dwarakanath Chinnaswamy Doraiswamy ${ }^{3}$
}

${ }_{1}^{1}$ Professor, Department of Periodontics and Implantology, Vishnu Dental College and Hospital, Bhimavaram, Andhra Pradesh. ${ }_{2}^{2}$ Post Graduate Student, Department of Periodontics and Implantology, Vishnu Dental College and Hospital, Andhra Pradesh. 3 Professor and HOD, Department of Periodontics and Implantology, Vishnu Dental College and Hospital, Bhimavaram, Andhra Pradesh.

\section{ABSTRACT}

\section{CONTEXT}

The harmony and beauty of a smile are characterized by both our teeth and gums, wherein the gingival display in the maxillary anterior teeth is more apparent than that of the posterior teeth. Anatomical characteristics of the periodontium, such as width of keratinized tissue and gingival thickness are known to reflect the health of the periodontium. Hence, it is cardinal to establish a relationship between the gingival parameters like the width of keratinized gingiva and the thickness of gingiva with the periodontal status in the anterior teeth region.

\section{AIM}

The objective of the study is to evaluate whether there is a positive correlation between the gingival thickness, width of the zone of gingival keratinized tissue and periodontal status.

\section{SETTINGS AND DESIGN}

This is a comparative clinical study with a sample size of 120 , of which 60 patients having periodontitis in the maxillary and mandibular anterior teeth regions were regarded as cases and 60 patients without any form of periodontitis in the maxillary and mandibular anterior teeth regions were regarded as controls.

\section{METHODS AND MATERIALS}

In all subjects, periodontal parameters like plaque index, gingival index, bleeding index, probing depth, clinical attachment level, width of keratinized gingiva and the number of recessions were recorded. The thickness of gingiva was recorded using an endodontic spreader and digital calipers.

\section{STATISTICAL ANALYSIS}

Karl Pearson correlation coefficient and unpaired t test were used for statistical analysis.

\section{RESULTS}

Among the cases, plaque index and gingival index negatively correlated with the width of keratinized tissue, the width of keratinized tissue positively correlated with thickness of gingiva and the probing depth, clinical attachment level and gingival recessions were negatively correlated with the width of keratinized tissue. The control group showed a positive correlation between the width of keratinized tissue, the probing depth and clinical attachment level.

\section{CONCLUSION}

There exists a difference in the width of keratinized tissue and gingival thickness among periodontitis patients and controls. Moreover, periodontal parameters like probing depth, clinical attachment level and incidence of gingival recession correlate with the width of keratinized gingiva and gingival tissue thickness in the anterior teeth region.

\section{KEYWORDS}

Keratinized Tissue Width, Gingival Thickness, Periodontal Status, Anterior Teeth.

HOW TO CITE THIS ARTICLE: Penmetsa GS, Supriya MS, Doraiswamy DC. Correlation of width of zone of keratinized tissue and gingival tissue thickness with periodontal status in anterior teeth. J. Evolution Med. Dent. Sci. 2016;5(47):2976-2979, DOI: $10.14260 /$ jemds/2016/694

Financial or Other, Competing Interest: None.

Submission 07-04-2016, Peer Review 11-05-2016,

Acceptance 17-05-2016, Published 11-06-2016.

Corresponding Author:

Dr. Marella Sai Supriya,

III MDS, Department of Periodontics and Implantology,

Vishnu Dental College and Hospital,

Vishnupur,

Bhimavaram-534202,

W. G. District,

Andhra Pradesh, India.

E-mail: msaisupriya@gmail.com

DOI: $10.14260 /$ jemds/2016/694

\section{INTRODUCTION}

The harmony and beauty of a smile is characterized by both our teeth and gums, wherein the soft tissue frame work around the teeth forms an integral part of overall dental aesthetics. An individual's perception of minor deviations from the ideal gingival margin is subjective, although the perception of gingival aesthetics is more objective than that of the dental aesthetics. Nevertheless, as the gingival display in the maxillary anterior teeth is more apparent than that of the posterior teeth, the pink aesthetics, i.e. gingival tissues form the soft tissue frame of the dentition and play an important role in dental aesthetics in the anterior region of the mouth. 
Hence, it is truly said that gingival and periodontal health around the teeth, especially the anteriors, is a major prerequisite for an aesthetic outlook of an individual.

Anatomical characteristics of the periodontium such as gingival thickness are known to reflect the health of the periodontium. Adequate zone of keratinized gingiva and thickness of attached gingiva are thought to be essential in order to maintain periodontal health and prevent the easy progression of periodontal disease. Lang NP and Loe $\mathrm{H}$ concluded that a width of at least $2 \mathrm{~mm}$ of keratinized mucosa, of which $1 \mathrm{~mm}$ was to be attached is cardinal for maintenance of normal periodontal health. ${ }^{1}$ However, certain other authors have proven that despite the presence of almost no attached gingiva, gingival health could be maintained.2,3,4 Hence, it is imperative to gain a greater knowledge of role of width of keratinized gingiva in the maintenance of a normal periodontium.

Gingival thickness has been shown to vary among different age groups, positioning of teeth within the arch and genetic factors. Some studies demonstrated that individuals with a thin phenotype had slightly more recession than subjects with wide and thick gingival tissues.5,6 As the gingival and periodontal health form a basic prerequisite for an aesthetic gingival morphology, the proper assessment of gingival aesthetics can only be performed under inflammation-free periodontal conditions. Unfortunately, not enough attention is paid in evaluation of the qualitative nature of the gingiva by a clinician during the course of formulating the treatment plan.

Therefore, it is cardinal to establish a relationship between the gingival parameters like the width of keratinized gingiva and the thickness of gingiva with the periodontal status in the anterior teeth region. Although there are studies correlating the width of keratinized gingiva and the thickness of gingiva independently, the present study is the first of its kind to correlate the width of keratinized gingiva and the thickness of gingiva with periodontal parameters in the anterior region.

\section{OBJECTIVE}

The objective of the study is to evaluate whether there is a positive correlation between the gingival thickness, width of the zone of gingival keratinized tissue and periodontal status.

\section{Inclusion Criteria}

Subjects of 20 to 65 years of age group who were systemically healthy with all the maxillary and mandibular anterior teeth present were included in the study.

\section{Exclusion Criteria}

Patients who were under the usage of drugs known to increase risk of gingival enlargement such as calcium-channel blockers, cyclosporine A or phenytoin, patients with severe anterior teeth crowding, patients having artificial crown restorations of the anteriors and patients showing any history of oral surgery in anterior teeth were excluded from the study.

\section{Clinical Parameters}

All the patients were anaesthetized in the upper and lower anterior teeth regions with a $2 \%$ lignocaine with adrenaline solution. The following measurements were taken on each maxillary and mandibular anterior tooth: Plaque Index (PI) by Silness and Loe 1964 and Gingival Index (GI) by Loe and Silness 1963.
Width of Keratinized Gingiva (WKG) was measured as the distance from the most coronal point on the gingival margin to the mucogingival junction, measured at the midpoint of the facial surface of the tooth. Probing Depth (PD), was measured on the mid-facial aspect from the gingival margin to the base of the sulcus/periodontal pocket and clinical attachment level was measured from the Cemento-Enamel Junction (CEJ) to the base of the sulcus/pocket on the mid-facial aspect. All the above three parameters were measured using a standard UNC15 periodontal probe.

On the other hand, gingival thickness was measured using an endodontic finger spreader attached to a rubber stopper and with a digital calliper. The endodontic probe was pierced at the centre of mid-facial aspect of gingiva, stopper was placed and measured using a digital calliper (Mitutoyo) of $0.01 \mathrm{~mm}$ resolution. The number of gingival recessions present in either of the anterior teeth regions was recorded.

\section{Statistical Analysis}

All the periodontal parameters including plaque index, gingival index, probing depth, clinical attachment level and number of gingival recessions were compared with the mean width of keratinized gingiva and thickness of attached gingiva using Karl Pearson correlation coefficient within the groups. Additionally, the mean widths of keratinized gingiva and thickness of attached gingiva among cases and controls were compared using unpaired t test.

\section{RESULTS}

A total of 120 subjects were included in the study, out of which 68 were males and 52 were females. The results were such that, in the cases group, plaque index and gingival index negatively correlated with the width of keratinized tissue with a Karl Pearson's correlation coefficient of -0.006 ( $p$ value 0.962 ) and -0.063 ( $p$ value 0.635 ) respectively, whereas the control group showed a positive correlation with the width of keratinized tissue with a Karl Pearson's correlation coefficient of 0.326 ( $p$ value of 0.011 ) and 0.063 ( $p$ value of 0.5 ), respectively (Table 1 ).

Width of keratinized tissue positively correlated with thickness of gingiva with a Pearson correlation of 0.252 ( $p$ value of 0.053 ) and 0.028 ( $p$ value of 0.833 ) in cases and controls, respectively. Similarly, the thickness of gingiva positively correlated with width of keratinized tissue with Pearson correlation values of 0.252 ( $p$ value of 0.053 ) and 0.028 ( $\mathrm{p}$ value of 0.833 ) in cases and controls, respectively (Table 1).

In cases, the probing depth, clinical attachment level and gingival recessions were negatively correlated with the width of keratinized tissue with a Karl Pearson's correlation coefficients of -0.016 ( $p$ value 0.903 ), -0.007 ( $p$ value 0.956 ) and -0.314 ( $p$ value 0.015 ), respectively. On the other hand, in controls, the probing depth and clinical attachment level showed a positive correlation with the width of keratinized tissue with a Karl Pearson's correlation coefficients of 0.036 (p value 0.785 ) and 0.035 ( $\mathrm{p}$ value 0.791 ) (Table 1 ).

In cases, the probing depth, clinical attachment level were negatively correlated with the thickness of attached gingiva with a Karl Pearson's correlation coefficients of 0.157 ( $p$ value 0.230 ), 0.221 ( $\mathrm{p}$ value 0.090), respectively and gingival recession showed a negative correlation with Karl Pearson's correlation coefficients of -0.06 ( $p$ value 0.649 ). 
While, in controls, probing depth and clinical attachment level showed a positive correlation with a Karl Pearson's correlation coefficient of 0.079 ( $p$ value 0.548 ) and 0.078 (p value 0.553 ), respectively (Table 1 ).
Also, the differences between mean values of the width of keratinized tissue and thickness of attached gingiva among controls and cases were 0.83 (SD 0.36) and 0.15 (SD 0.07), respectively, which were statistically significant (Table 2 ).

\begin{tabular}{|c|c|c|c|c|c|}
\hline \multicolumn{6}{|c|}{ Correlations Analysis } \\
\hline \multirow{2}{*}{ Variables } & \multirow{2}{*}{ Formula } & \multicolumn{2}{|c|}{ Cases } & \multicolumn{2}{|c|}{ Controls } \\
\hline & & W.KG & GT & W.KG & GT \\
\hline \multirow{2}{*}{ PI } & Pearson Correlation & -0.006 & 0.071 & 0.326 & 0.089 \\
\hline & P value & 0.962 & 0.592 & $0.011 \mathrm{~S}$ & 0.500 \\
\hline \multirow{2}{*}{ GI } & Pearson Correlation & -0.063 & 0.033 & 0.063 & -0.094 \\
\hline & P value & 0.635 & 0.804 & 0.634 & 0.477 \\
\hline \multirow{2}{*}{ W.KG } & Pearson Correlation & 1 & 0.252 & 1.000 & 0.028 \\
\hline & P value & & 0.053 & & 0.833 \\
\hline \multirow{2}{*}{ GT } & Pearson Correlation & 0.252 & 1 & 0.028 & 1.000 \\
\hline & P value & 0.053 & & 0.833 & \\
\hline \multirow{2}{*}{ PD } & Pearson Correlation & -0.016 & 0.157 & 0.036 & 0.079 \\
\hline & P value & 0.903 & 0.230 & 0.785 & 0.548 \\
\hline \multirow{2}{*}{ CAL } & Pearson Correlation & -0.007 & 0.221 & 0.035 & 0.078 \\
\hline & P value & 0.956 & 0.090 & 0.791 & 0.553 \\
\hline \multirow{2}{*}{ GR } & Pearson Correlation & -0.314 & -0.06 & -- & -- \\
\hline & P value & $0.015 \mathrm{~S}$ & 0.649 & -- & -- \\
\hline & & Table 1 & & & \\
\hline
\end{tabular}

Statistical Analysis: Karl Pearson's correlation coefficient. Statistically significant if $\mathrm{P}<0.05$

\begin{tabular}{|c|c|c|c|c|c|c|c|c|}
\hline \multirow{2}{*}{ Variables } & \multicolumn{2}{|c|}{ Cases } & \multicolumn{2}{c|}{ Controls } & \multicolumn{2}{c|}{ Difference } & \multirow{2}{*}{ T value } & \multirow{2}{*}{ P value } \\
\cline { 2 - 8 } & Mean & SD & Mean & SD & Mean & SD & & $0.000 \mathrm{~S}$ \\
\hline W.KG & 3.32 & 0.93 & 4.15 & 1.29 & 0.83 & 0.36 & 4.215 & $0.003 \mathrm{~S}$ \\
\hline GT & 0.70 & 0.23 & 0.85 & 0.30 & 0.15 & 0.07 & 3.047 & 0 \\
\hline \multicolumn{9}{|c|}{ Table 2 } \\
\hline
\end{tabular}

Statistical Analysis: Unpaired t test. Statistically significant if $\mathrm{P}<0.05$

\section{DISCUSSION}

In recent years the dimensions of different parts of the masticatory mucosa, especially the keratinized gingiva, has become a subject of considerable interest in periodontics both from an epidemiologic and a therapeutic point of view. Since studies have concluded that the thickness of the gingiva plays a vital role in development of mucogingival problems and in the success of treatment for recession, assessment of gingival thickness is relevant to clinical periodontics. It has been suggested that a certain width of attached, keratinized gingiva is necessary for gingival health., 7 Thus, keratinized gingiva may be more suitable to withstand the trauma of mastication and tooth brushing than unkeratinized alveolar mucosa.9,10 Hence, the present study was undertaken to demonstrate the correlations between the width and thickness of the keratinized gingival tissue with the periodontal status.

In this study, a positive correlation was observed between the width of the zone of gingival keratinized tissue and gingival thickness in both cases and controls. Similar to the findings of the present study, several periodontal biotype and phenotype classifications have endeavoured to positively correlate gingival thickness with the width of the zone of gingival keratinized tissue. In a cluster analysis study three clusters A1, A2 and B were observed, where A1 cluster subjects showed a narrow band of gingiva of about $3.5 \mathrm{~mm}$, whereas gingiva of cluster A2 subjects was considerably wider $(4.27 \pm 0.80 \mathrm{~mm}$ at canines up to $5.13 \pm 0.67 \mathrm{~mm}$ at lateral incisors) and cluster $B$ had considerably thicker $(0.81 \pm 0.16 \mathrm{~mm}$ at canines up to $1.29 \pm 0.30 \mathrm{~mm}$ at central incisors) and wider gingiva $(5.09 \pm 1.00 \mathrm{~mm}$ at canines up to $6.59 \pm 0.58 \mathrm{~mm}$ at lateral incisors). ${ }^{5}$ In another cluster analysis study, similar groups were identified, i.e. a cluster had a significantly thicker and wider gingiva, while another cluster showed normal gingival thickness but a narrow zone of keratinized tissue. ${ }^{11}$

The present study demonstrated that the plaque and gingival indices showed a negative correlation with the width of keratinized tissue among the cases. This finding is in agreement with that of a study conducted by Lang and Loe, who demonstrated that areas of minimum width of attached gingiva may by their increased mobility facilitate subgingival plaque deposition. ${ }^{1}$, whereas the control group showed a positive correlation with the width of keratinized tissue and thickness of gingiva.

In the present study, the number of gingival recession increased with a decrease in width of keratinized gingiva and this correlation was statistically significant. This is in agreement with a few cross-sectional studies showing a correlation between the frequency of recessions and the width of the gingiva as mentioned in the reviews by Hall and Wennstrom.12,13 However, later, some of the longitudinal studies demonstrated that the lack of or the presence of minimal amounts of attached gingiva at the buccal aspects of teeth do not necessarily result in the development of soft tissue recessions. ${ }^{14,15,16,17} \mathrm{~A}$ retrospective study conducted by Lindhe and Nyman have shown evidences in alterations of the position of the marginal soft tissue following periodontal surgery. Also, it was concluded that in the presence of proper plaque control measures, the apico-coronal width of gingiva and the presence or absence of an attached portion of gingiva are not of decisive significance for the maintenance. ${ }^{18}$ 
In our study, the incidence of gingival recession also increased with a decrease in thickness of gingiva, the findings of which are not significant. This was similar to the results seen in previous study, which showed trends of more recession in subjects with thin gingiva which was also not significant. ${ }^{5}$

In the present study the periodontal probing depth was associated with thicker gingiva among the cases, which was in accordance with observations made by several other authors who also reported that subjects with a thick periodontal phenotype had on an average higher mean periodontal probing depth. ${ }^{19,20,21}$ However, such a correlation between the probing depth and gingival thickness did not exist in the control group.

\section{CONCLUSION}

Within the limitations of this study, it was concluded that there definitely exists a difference in the width of keratinized tissue and gingival thickness among periodontitis patients and controls. Moreover, it has been postulated that periodontal parameters like probing depth, clinical attachment level and incidence of gingival recession correlate with the width of keratinized gingiva and gingival tissue thickness in the anterior teeth region. As a thorough and detailed information on this aspect is still not evident in the literature, further studies are warranted to evaluate the correlation between the gingival thickness, width of the zone of gingival keratinized tissue and periodontal status of overall dentition.

\section{REFERENCES}

1. Lang N, Löe H. The relationship between the width of keratinized gingiva and gingival health. J Periodontol 1972;43(10):623-7.

2. Dorfman HS, Kennedy JE, Bird WC. Longitudinal evaluation of free autogenous gingival grafts. J Clin Periodontol 1980;7(4):316-24.

3. Miyasato M, Crigger M, Egelberg J. Gingival condition in areas of minimal and appreciable width of keratinized gingiva. J Clin Periodontol 1977;4(3):200-209.

4. Wennstrom J, Lindhe J. Role of attached gingiva for maintenance of periodontal health. J Clin Periodontol 1983;10(2):206-21.

5. Müller HP, Heinecke A, Schaller N, et al. Masticatory mucosa in subjects with different periodontal phenotypes. J Clin Periodontol 2000;27(9):621-6.

6. Kitchin PC. The prevalence of tooth root exposure and the relation of the extent of such exposure to the degree of abrasion in different age classes. J Dent Research 1941;20:565-81.
7. Bowers GM. A study of the width of attached gingiva. J Periodontol 1963;34:201-9.

8. Ainamo J, Löe H. Anatomical characteristics of gingiva. A clinical and microscopic study of the free and attached gingiva. J Periodontol 1966;37(1):5-13.

9. Kao RT, Pasquinelli K. Thick vs. thin gingival tissue: a key determinant in tissue response to disease and restorative treatment. J Calif Dent Assoc 2002;30(7):521-6.

10. Maynard JG, Wilson RD. Diagnosis and management of mucogingival problems in children. Dent Clin North Am 1980;24(4):683-703.

11. Müller HP, Eger T. Gingival phenotypes in young male adults. J Clin Periodontol 1997;24(1):65-71.

12. Hall WB. The current status of mucogingival problems and their therapy. J Periodontol 1981;52(9):569-75.

13. Wennstrom JL. Keratinized and attached gingivaregenerative potential and significance for periodontal health. Thesis. University of Gothenburg, Gothenburg, Sweden 1982.

14. Wennstrom JL. Lack of association between width of attached gingiva and development of soft tissue recession. A 5-year longitudinal study. J Clin Periodontol 1987;14(3):181-4.

15. Kennedy JE, Bird WC, Palcanis KG, et al. A longitudinal evaluation of varying widths of attached gingiva. J Clin Periodontol 1985;12(8):667-75.

16. Schoo WH, van der Velden U. Marginal soft tissue recessions with and without attached gingiva. J Periodont Res 1985;20(2):209-11.

17. Kisch J, Badersten A, Egelberg J. Longitudinal observation of unattached, mobile gingival areas. J Clin Periodontol 1986;13(2):131-4.

18. Lindhe J, Nyman S. Alterations of the position of the marginal soft tissue following periodontal surgery. Journal of Clinical Periodontology 1980;7(6):525-30.

19. Muller HP, Kononen E. Variance components of gingival thickness. J Periodont Res 2005;40(3):239-44.

20. Eger T, Muller HP, Heinecke A. Ultrasonic determination of gingival thickness. Subject variation and influence of tooth type and clinical features. J Clin Periodontol 1996;23(9):839-45.

21. Olsson M, Lindhe J, Marinello CP. On the relationship between crown form and clinical features of the gingiva in adolescents. J Clin Periodontol 1993;20(8):570-7. 